\title{
THE PLATEAU-BÉZIER PROBLEM WITH WEAK-AREA FUNCTIONAL*
}

\author{
Yongxia Hao \\ Faculty of Science, Jiangsu University, Zhenjiang 212013, China \\ Email: yongxiahaoujs@ujs.edu.cn
}

\begin{abstract}
In this paper, we present a new method to solve the Plateau-Bézier problem. A new energy functional called weak-area functional is proposed as the objective functional to obtain the approximate minimal Bézier surface from given boundaries. This functional is constructed based on Dirichlet energy and weak isothermal parameterization condition. Experimental comparisons of the weak-area functional method with existing Dirichlet, quasi-harmonic, the strain energy-minimizing, harmonic and biharmonic masks are performed which show that the weak-area functional method are among the best by choosing appropriate parameters.
\end{abstract}

Mathematics subject classification: 65D17, 65D18.

Key words: Minimal surface, Plateau-Bézier problem, Weak isothermal parameterization, Weak-area functional.

\section{Introduction}

The problem of finding a surface that minimizes the area with prescribed border is called the Plateau problem $[3,4,15,16]$. Such surfaces are called minimal surfaces and characterized by the fact that the mean curvature vanishes. The minimal surface has attracted scientists for many years and has been studied extensively in many literatures, such as $[5,12,13,21-23]$. Part of the interest stems from the fact that it is so easily realizable physically in the form of soap films, and for this reason it has been studied not only mathematically, but also physically for many years [19]. The fascinating characters of minimal surface make it to be widely used in many areas such as architecture, material science, ship manufacture, biology and so on [17]. For instance, architecture inspired from minimal surface embodies the unite of economy and beauty. Furthermore, scientists and engineers have anticipated the nanotechnology applications of minimal surface in areas of molecular engineering and materials science [20]. Applications of minimal surface in aesthetic design have also been presented in [18].

As we know, only a few minimal surfaces have been found in closed form. Hence, numerical methods have been devised to construct approximate minimal surface. Brakke proposed an approach to compute a parametric minimal surface with the finite element method [1]. Direct simulation of surface tension forces on a grid of marker particles is used for the minimal surface approximation in $[2,9]$. Jung et al. proposed a variational level set approach for the surface area minimization of triply-periodic surfaces [10]. Tråsdahl and Rønquist presented an algorithm for finding high order numerical approximations of minimal surfaces with a fixed boundary [19].

In order to find an approximate Bézier solution of the Plateau problem, J. Monterde proposed the Plateau-Bézier problem [12], which is to find the surface of minimal area from among

\footnotetext{
* Received February 26, 2019 / Accepted June 20, 2019 /
}

Published online September 18, 2019 / 
all Bézier surfaces with given boundary curves. Because of the high nonlinearity of the area functional, several energy functionals are used to approximate the area functional, which lead to easy management for the Plateau-Bézier problem. The first one is the Dirichlet functional employed as a replacement to solve the Plateau-Bézier problem in [12]. Based on this functional, a multiresolution analysis method with B-splines is proposed to obtain the parametric surface of minimal area in [7]. Moreover, the minimal quasi-Bézier surfaces in non-polynomial space are also investigated by the Dirichlet method and harmonic method in [8]. A new energy functional called quasi-harmonic energy functional is proposed in [21] as the objective functional to obtain the quasi-harmonic Bézier surface from given boundaries. Bending energy functional [11] and mean curvature energy functional [24] are also used for approximating the solution of the Plateau-Bézier problem.

Harmonic surface is related to minimal surface. The corresponding Euler-Lagrange equation of the Dirichlet functional is $\Delta \mathbf{r}=0$, which defines the harmonic surface. Therefore, harmonic Bézier surface and biharmonic Bézier surface are also proposed as an approximation solution of the Plateau-Bézier problem [14]. A surface with isothermal parameterization is minimal surface if and only if it is harmonic surface. This is exactly the theoretical basis of the Dirichlet functional and quasi-harmonic functional to replace the area functional. However, both these two functionals are constructed without any thought of the isothermal parameterization. Therefore in this paper, we introduce a new energy functional constructed based on Dirichlet functional and isothermal parameterization to solve the Plateau-Bézier problem.

The remainder of this paper is organized as follows. Some preliminaries and weak-area energy functional are introduced in Section 2. Section 3 presents the sufficient and necessary conditions for Bézier surfaces with minimal weak-area energy. Some comparisons among different methods are presented in Section 4. Finally, we conclude and list some future works in Section 5 .

\section{Preliminary and Weak-Area Functional}

In this section, we shall review some concepts and results related to minimal surfaces $[15,16]$, and introduce the weak isothermal parameterization and weak-area functional.

\subsection{Preliminary}

For a parametric surface $\mathbf{r}(u, v)$, the coefficients of the first fundamental form are

$$
E=\left\langle\mathbf{r}_{u}, \mathbf{r}_{u}\right\rangle, \quad F=\left\langle\mathbf{r}_{u}, \mathbf{r}_{v}\right\rangle, \quad G=\left\langle\mathbf{r}_{v}, \mathbf{r}_{v}\right\rangle,
$$

where $\mathbf{r}_{u}, \mathbf{r}_{v}$ are the first-order partial derivatives of $\mathbf{r}(u, v)$ with respect to $u$ and $v$ respectively, and $\langle$,$\rangle defines the dot product of the vectors. The coefficients of the second fundamental form$ of $\mathbf{r}(u, v)$ are

$$
L=\left(\mathbf{r}_{u}, \mathbf{r}_{v}, \mathbf{r}_{u u}\right), \quad M=\left(\mathbf{r}_{u}, \mathbf{r}_{v}, \mathbf{r}_{u v}\right), \quad N=\left(\mathbf{r}_{u}, \mathbf{r}_{v}, \mathbf{r}_{v v}\right),
$$

where $\mathbf{r}_{u u}, \mathbf{r}_{v v}$ and $\mathbf{r}_{u v}$ are the second-order partial derivatives of $\mathbf{r}(u, v)$ and $(,$,$) defines the$ mixed product of the vectors. Then the mean curvature $H$ and the Gaussian curvature $K$ of $\mathbf{r}(u, v)$ are

$$
H=\frac{E N-2 F M+L G}{2\left(E G-F^{2}\right)}, \quad K=\frac{L N-M^{2}}{E G-F^{2}} .
$$


Definition 2.1. If $\mathbf{r}(u, v)$ satisfies $E=G, F=0$, then $\mathbf{r}(u, v)$ is called surface with isothermal parameterizations.

Definition 2.2. If $\mathbf{r}(u, v)$ satisfies $H=0$, then $\mathbf{r}(u, v)$ is called minimal surface.

Theorem 2.1. The surface with isothermal parameter is a minimal surface if and only if it is a harmonic surface.

\subsection{Weak-area functional}

Since the isothermal parameterization condition is too strong to satisfy for a parametric surface $\mathbf{r}(u, v)$, and it may impose too many restrictions on the resulting surface, from Definition 2.1 , we introduce the following weak isothermal parameterization.

Definition 2.3. If $\mathbf{r}(u, v)$ satisfies

$$
\int_{\Omega}(E-G) d u d v=0, \quad \int_{\Omega} F d u d v=0,
$$

then $\mathbf{r}(u, v)$ is called a surface with weak isothermal parameterizations.

Clearly, the weak isothermal parameterization condition is defined on the whole surface, while the isothermal parameterization is on every point of the surface. Moreover, a surface with isothermal parameterization satisfies naturely the weak isothermal parameterization condition.

Due to the high nonlinearity of area functional, the Dirichlet functional $D(\mathbf{r})$ is commonly used instead:

$$
D(\mathbf{r})=\frac{1}{2} \int_{\Omega}(E+G) d u d v .
$$

Moreover, the area and the Dirichlet functional are equal only if $E=G, F=0$, i.e., for isothermal patches. Based on this point, we propose a new energy functional $W(\mathbf{r})$ as objective functional to solve the Plateau-Bézier problem:

$$
W(\mathbf{r})=\frac{1}{2} \int_{\Omega}(E+G) d u d v+\lambda\left(\int_{\Omega}(E-G) d u d v+\int_{\Omega} F d u d v\right),
$$

where $\lambda$ is a parameter balancing the Dirichlet functional and the weak isothermal parameterization condition. In this paper, $W(\mathbf{r})$ is called weak-area functional. Obviously, the weak-area functional is defined as a linear combination of Dirichlet energy and weak isothermal parameterization.

Since the weak isothermal parameterization condition could be positive or negative, here we restrict $\lambda \in[-1,1]$ to ensure that $\frac{1}{2}(E+G)+\lambda(E-G)+\lambda F \geq 0$ and the extremal of $W(\mathbf{r})$ exists. When $\lambda$ is set to zero, then $W(\mathbf{r})$ is just a Dirichlet one. Once the value of $\lambda$ is determined, all the corresponding inner control points can be obtained by directly solving a system of linear equations as the Dirichlet method does.

\section{Extremals of the Weak-Area Functional}

In this section, we will present the sufficient and necessary condition for the extremals of the weak-area functional. Let us say we are not computing the Euler-Lagrange equations of the functional, but compute the surface where the gradient of the functional vanishes. 
Theorem 3.1. Given the boundary control points $\left\{\mathbf{P}_{0 j}, \mathbf{P}_{n j}\right\}$ and $\left\{\mathbf{P}_{i 0}, \mathbf{P}_{i m}\right\}$ of a tensor product Bézier surface $\mathbf{r}(u, v)=\sum_{i=0}^{n} \sum_{j=0}^{m} \mathbf{P}_{i j} B_{i}^{n}(u) B_{j}^{m}(v)$, then $\mathbf{r}(u, v)$ is the extremal of the weak-area functional $W(\mathbf{r})$ if and only if the inner control points $\left\{\mathbf{P}_{i j}\right\}_{i, j=1}^{n-1, m-1}$ satisfy

$$
\begin{aligned}
& \sum_{k=0}^{n-1} \sum_{l=0}^{m} \Delta^{1,0} \mathbf{P}_{k l}\left(\frac{(2 \lambda+1) n^{2}}{(2 n-1)(2 m+1)}\left(A_{i-1, k}^{n-1}-A_{i, k}^{n-1}\right) A_{j, l}^{m}+\frac{\lambda}{4}\left(A_{j-1, l}^{m-1, m}-A_{j, l}^{m-1, m}\right) A_{i, k}^{n, n-1}\right) \\
& \quad+\sum_{k=0}^{n} \sum_{l=0}^{m-1} \Delta^{0,1} \mathbf{P}_{k l}\left(\frac{(1-2 \lambda) m^{2}}{(2 n+1)(2 m-1)}\left(A_{j-1, l}^{m-1}-A_{j, l}^{m-1}\right) A_{i, k}^{n}+\frac{\lambda}{4}\left(A_{i-1, k}^{n-1, n}-A_{i, k}^{n-1, n}\right) A_{j, l}^{m, m-1}\right)=0
\end{aligned}
$$

where

$$
A_{i, j}^{n, m}=\frac{\left(\begin{array}{c}
n \\
i
\end{array}\right)\left(\begin{array}{c}
m \\
j
\end{array}\right)}{\left(\begin{array}{c}
n+m \\
i+j
\end{array}\right)}, \quad A_{i, j}^{n}=A_{i, j}^{n, n} .
$$

Proof. Let us compute the gradient of the weak-area functional with respect to the coordinates of a inner control point $\mathbf{P}_{i j}=\left(x_{i j}^{1}, x_{i j}^{2}, x_{i j}^{3}\right)$. For any $a \in\{1,2,3\}, i \in\{1, \ldots, n-1\}, j \in$ $\{1, \ldots, m-1\}$,

$$
\frac{\partial W(\mathbf{r})}{\partial x_{i j}^{a}}=\left(\frac{1}{2}+\lambda\right) \int_{\Omega} \frac{\partial E}{\partial x_{i j}^{a}} d u d v+\left(\frac{1}{2}-\lambda\right) \int_{\Omega} \frac{\partial G}{\partial x_{i j}^{a}} d u d v+\lambda \int_{\Omega} \frac{\partial F}{\partial x_{i j}^{a}} d u d v .
$$

Note that

$$
\mathbf{r}_{u}=n \sum_{k=0}^{n-1} \sum_{l=0}^{m} B_{k}^{n-1}(u) B_{l}^{m}(v) \Delta^{1,0} \mathbf{P}_{k l}, \mathbf{r}_{v}=m \sum_{k=0}^{n} \sum_{l=0}^{m-1} B_{k}^{n}(u) B_{l}^{m-1}(v) \Delta^{0,1} \mathbf{P}_{k l} .
$$

Then we have

$$
\begin{aligned}
\frac{\partial \mathbf{r}_{u}}{\partial x_{i j}^{a}} & =n\left(B_{i-1}^{n-1}(u)-B_{i}^{n-1}(u)\right) B_{j}^{m}(v) \mathbf{e}^{a}, \\
\frac{\partial \mathbf{r}_{v}}{\partial x_{i j}^{a}} & =m B_{i}^{n}(u)\left(B_{j-1}^{m-1}(v)-B_{j}^{m-1}(v)\right) \mathbf{e}^{a} \\
\frac{\partial E}{\partial x_{i j}^{a}} & =2\left(\mathbf{r}_{u}, \frac{\partial \mathbf{r}_{u}}{\partial x_{i j}^{a}}\right)=2 n^{2} \sum_{k=0}^{n-1} \sum_{l=0}^{m}\left(B_{i-1}^{n-1}(u)-B_{i}^{n-1}(u)\right) B_{k}^{n-1}(u) B_{j}^{m}(v) B_{l}^{m}(v)\left(\Delta^{1,0} \mathbf{P}_{k l}, \mathbf{e}^{a}\right), \\
\frac{\partial G}{\partial x_{i j}^{a}} & =2\left(\mathbf{r}_{v}, \frac{\partial \mathbf{r}_{v}}{\partial x_{i j}^{a}}\right)=2 m^{2} \sum_{k=0}^{n} \sum_{l=0}^{m-1} B_{i}^{n}(u) B_{k}^{n}(u)\left(B_{j-1}^{m-1}(v)-B_{j}^{m-1}(v)\right) B_{l}^{m-1}(v)\left(\Delta^{0,1} \mathbf{P}_{k l}, \mathbf{e}^{a}\right), \\
\frac{\partial F}{\partial x_{i j}^{a}} & =\left(\frac{\partial \mathbf{r}_{u}}{\partial x_{i j}^{a}}, \mathbf{r}_{v}\right)+\left(\mathbf{r}_{u}, \frac{\partial \mathbf{r}_{v}}{\partial x_{i j}^{a}}\right) \\
& =m n\left(\sum_{k=0}^{n} \sum_{l=0}^{m-1}\left(B_{i-1}^{n-1}(u)-B_{i}^{n-1}(u)\right) B_{k}^{n}(u) B_{j}^{m}(v) B_{l}^{m-1}(v)\left(\Delta^{0,1} \mathbf{P}_{k l}, \mathbf{e}^{a}\right)\right. \\
& \left.+\sum_{k=0}^{n-1} \sum_{l=0}^{m} B_{i}^{n}(u) B_{k}^{n-1}(u)\left(B_{j-1}^{m-1}(v)-B_{j}^{m-1}(v)\right) B_{l}^{m}(v)\left(\Delta^{1,0} \mathbf{P}_{k l}, \mathbf{e}^{a}\right)\right)
\end{aligned}
$$

where $\mathbf{e}^{1}=(1,0,0), \mathbf{e}^{2}=(0,1,0), \mathbf{e}^{3}=(0,0,1)$.

Note that the Bernstein function has the following formulas:

$$
B_{i}^{n}(u) B_{j}^{m}(u)=\frac{\left(\begin{array}{c}
n \\
i
\end{array}\right)\left(\begin{array}{c}
m \\
j
\end{array}\right)}{\left(\begin{array}{c}
n+m \\
i+j
\end{array}\right)} B_{i+j}^{n+m}(u), \quad \int_{0}^{1} B_{i}^{n}(u) d u=\frac{1}{n+1} .
$$


By using the notation of (3.2), we obtain

$$
\begin{aligned}
\frac{\partial W(\mathbf{r})}{\partial x_{i j}^{a}}=\sum_{k=0}^{n-1} & \sum_{l=0}^{m}\left(\Delta^{1,0} \mathbf{P}_{k l}, \mathbf{e}^{a}\right)\left(\frac{(2 \lambda+1) n^{2}}{(2 n-1)(2 m+1)}\left(A_{i-1, k}^{n-1, n-1}-A_{i, k}^{n-1, n-1}\right) A_{j, l}^{m, m}\right. \\
& \left.+\frac{\lambda}{4}\left(A_{j-1, l}^{m-1, m}-A_{j, l}^{m-1, m}\right) A_{i, k}^{n, n-1}\right) \\
& +\sum_{k=0}^{n} \sum_{l=0}^{m-1}\left(\Delta^{0,1} \mathbf{P}_{k l}, \mathbf{e}^{a}\right)\left(\frac{(1-2 \lambda) m^{2}}{(2 n+1)(2 m-1)}\left(A_{j-1, l}^{m-1, m-1}-A_{j, l}^{m-1, m-1}\right) A_{i, k}^{n, n}\right. \\
& \left.+\frac{\lambda}{4}\left(A_{i-1, k}^{n-1, n}-A_{i, k}^{n-1, n}\right) A_{j, l}^{m, m-1}\right) .
\end{aligned}
$$

So fixing the boundary control points and taking the inner control points as unknowns, the linear system (3.1) is always compatible and can be solved in terms of the boundary control points.

If $n=m=2$, then there is just one equation corresponding to the inner control point $\mathbf{P}_{11}$.

Proposition 3.1. Given the boundary control points, the biquadratic Bézier surface is an extremal of the weak-area functional if and only if $\mathbf{P}_{11}$ satisfies the condition:

$$
\begin{aligned}
\mathbf{P}_{11}=-\frac{1}{8}(( & -3-5 \lambda) \mathbf{P}_{00}+(1-10 \lambda) \mathbf{P}_{01}+(5 \lambda-3) \mathbf{P}_{02}+(10 \lambda+1) \mathbf{P}_{10}+(10 \lambda+1) \mathbf{P}_{12} \\
& \left.+(5 \lambda-3) \mathbf{P}_{20}+(1-10 \lambda) \mathbf{P}_{21}+(-3-5 \lambda) \mathbf{P}_{22}\right)
\end{aligned}
$$

If $n=m=3$, there are four equations corresponding to the inner control points $\mathbf{P}_{11}, \mathbf{P}_{12}, \mathbf{P}_{21}, \mathbf{P}_{22}$.

Proposition 3.2. Given the boundary control points, the bicubic Bézier surface is an extremal of the weak-area functional if and only if

$$
\begin{aligned}
\mathbf{P}_{11}= & \frac{1}{22533 \lambda^{4}-207336 \lambda^{2}+121680}\left(\left(-58373 \lambda^{4}-145218 \lambda^{3}-15404 \lambda^{2}+128456 \lambda+74880\right) \mathbf{P}_{00}\right. \\
& +\left(-110257 \lambda^{4}-164008 \lambda^{3}+54344 \lambda^{2}+161696 \lambda-34320\right) \mathbf{P}_{01} \\
& +\left(-46816 \lambda^{4}+92168 \lambda^{3}-125008 \lambda^{2}-78496 \lambda+37440\right) \mathbf{P}_{02} \\
& +\left(37226 \lambda^{4}-39372 \lambda^{3}+38808 \lambda^{2}-37456 \lambda\right) \mathbf{P}_{03} \\
& +\left(85351 \lambda^{4}+239912 \lambda^{3}+47848 \lambda^{2}-232864 \lambda-34320\right) \mathbf{P}_{10} \\
& +\left(96292 \lambda^{4}+19703 \lambda^{3}-37754 \lambda^{2}+31364 \lambda+23400\right) \mathbf{P}_{13} \\
& +\left(114016 \lambda^{4}+30728 \lambda^{3}-191312 \lambda^{2}-63136 \lambda+37440\right) \mathbf{P}_{20} \\
& +\left(102424 \lambda^{4}+219332 \lambda^{3}-101768 \lambda^{2}-56464 \lambda-6240\right) \mathbf{P}_{23} \\
& +\left(52906 \lambda^{4}-55052 \lambda^{3}-95592 \lambda^{2}+96944 \lambda\right) \mathbf{P}_{30} \\
& +\left(-73360 \lambda^{4}+87023 \lambda^{3}+104150 \lambda^{2}-34396 \lambda+23400\right) \mathbf{P}_{31} \\
& +\left(-122584 \lambda^{4}-149308 \lambda^{3}+156728 \lambda^{2}+35696 \lambda-6240\right) \mathbf{P}_{32} \\
& \left.+\left(-54292 \lambda^{4}-135908 \lambda^{3}-42376 \lambda^{2}+48656 \lambda+6240\right) \mathbf{P}_{33}\right)
\end{aligned}
$$




$$
\begin{aligned}
& \mathbf{P}_{12}=\frac{1}{22533 \lambda^{4}-207336 \lambda^{2}+121680}\left(\left(52906 \lambda^{4}+55052 \lambda^{3}-95592 \lambda^{2}-96944 \lambda\right) \mathbf{P}_{00}\right. \\
& +\left(114016 \lambda^{4}-30728 \lambda^{3}-191312 \lambda^{2}+63136 \lambda+37440\right) \mathbf{P}_{01} \\
& +\left(85351 \lambda^{4}-239912 \lambda^{3}+47848 \lambda^{2}+232864 \lambda-34320\right) \mathbf{P}_{02} \\
& +\left(-58373 \lambda^{4}+145218 \lambda^{3}-15404 \lambda^{2}-128456 \lambda+74880\right) \mathbf{P}_{03} \\
& +\left(-73360 \lambda^{4}-87023 \lambda^{3}+104150 \lambda^{2}+34396 \lambda+23400\right) \mathbf{P}_{10} \\
& +\left(-110257 \lambda^{4}+164008 \lambda^{3}+54344 \lambda^{2}-161696 \lambda-34320\right) \mathbf{P}_{13} \\
& +\left(-122584 \lambda^{4}+149308 \lambda^{3}+156728 \lambda^{2}-35696 \lambda-6240\right) \mathbf{P}_{20} \\
& +\left(-46816 \lambda^{4}-92168 \lambda^{3}-125008 \lambda^{2}+78496 \lambda+37440\right) \mathbf{P}_{23} \\
& +\left(-54292 \lambda^{4}+135908 \lambda^{3}-42376 \lambda^{2}-48656 \lambda+6240\right) \mathbf{P}_{30} \\
& +\left(102424 \lambda^{4}-219332 \lambda^{3}-101768 \lambda^{2}+56464 \lambda-6240\right) \mathbf{P}_{31} \\
& +\left(96292 \lambda^{4}-19703 \lambda^{3}-37754 \lambda^{2}-31364 \lambda+23400\right) \mathbf{P}_{32} \\
& \left.+\left(37226 \lambda^{4}+39372 \lambda^{3}+38808 \lambda^{2}+37456 \lambda\right) \mathbf{P}_{33}\right), \\
& \mathbf{P}_{21}=\frac{1}{22533 \lambda^{4}-207336 \lambda^{2}+121680}\left(\left(37226 \lambda^{4}+39372 \lambda^{3}+38808 \lambda^{2}+37456 \lambda\right) \mathbf{P}_{00}\right. \\
& +\left(96292 \lambda^{4}-19703 \lambda^{3}-37754 \lambda^{2}-31364 \lambda+23400\right) \mathbf{P}_{01} \\
& +\left(102424 \lambda^{4}-219332 \lambda^{3}-101768 \lambda^{2}+56464 \lambda-6240\right) \mathbf{P}_{02} \\
& +\left(-54292 \lambda^{4}+135908 \lambda^{3}-42376 \lambda^{2}-48656 \lambda+6240\right) \mathbf{P}_{03} \\
& +\left(-46816 \lambda^{4}-92168 \lambda^{3}-125008 \lambda^{2}+78496 \lambda+37440\right) \mathbf{P}_{10} \\
& +\left(-122584 \lambda^{4}+149308 \lambda^{3}+156728 \lambda^{2}-35696 \lambda-6240\right) \mathbf{P}_{13} \\
& +\left(-110257 \lambda^{4}+164008 \lambda^{3}+54344 \lambda^{2}-161696 \lambda-34320\right) \mathbf{P}_{20} \\
& +\left(-73360 \lambda^{4}-87023 \lambda^{3}+104150 \lambda^{2}+34396 \lambda+23400\right) \mathbf{P}_{23} \\
& +\left(-58373 \lambda^{4}+145218 \lambda^{3}-15404 \lambda^{2}-128456 \lambda+74880\right) \mathbf{P}_{30} \\
& +\left(85351 \lambda^{4}-239912 \lambda^{3}+47848 \lambda^{2}+232864 \lambda-34320\right) \mathbf{P}_{31} \\
& +\left(114016 \lambda^{4}-30728 \lambda^{3}-191312 \lambda^{2}+63136 \lambda+37440\right) \mathbf{P}_{32} \\
& \left.+\left(52906 \lambda^{4}+55052 \lambda^{3}-95592 \lambda^{2}-96944 \lambda\right) \mathbf{P}_{33}\right),
\end{aligned}
$$

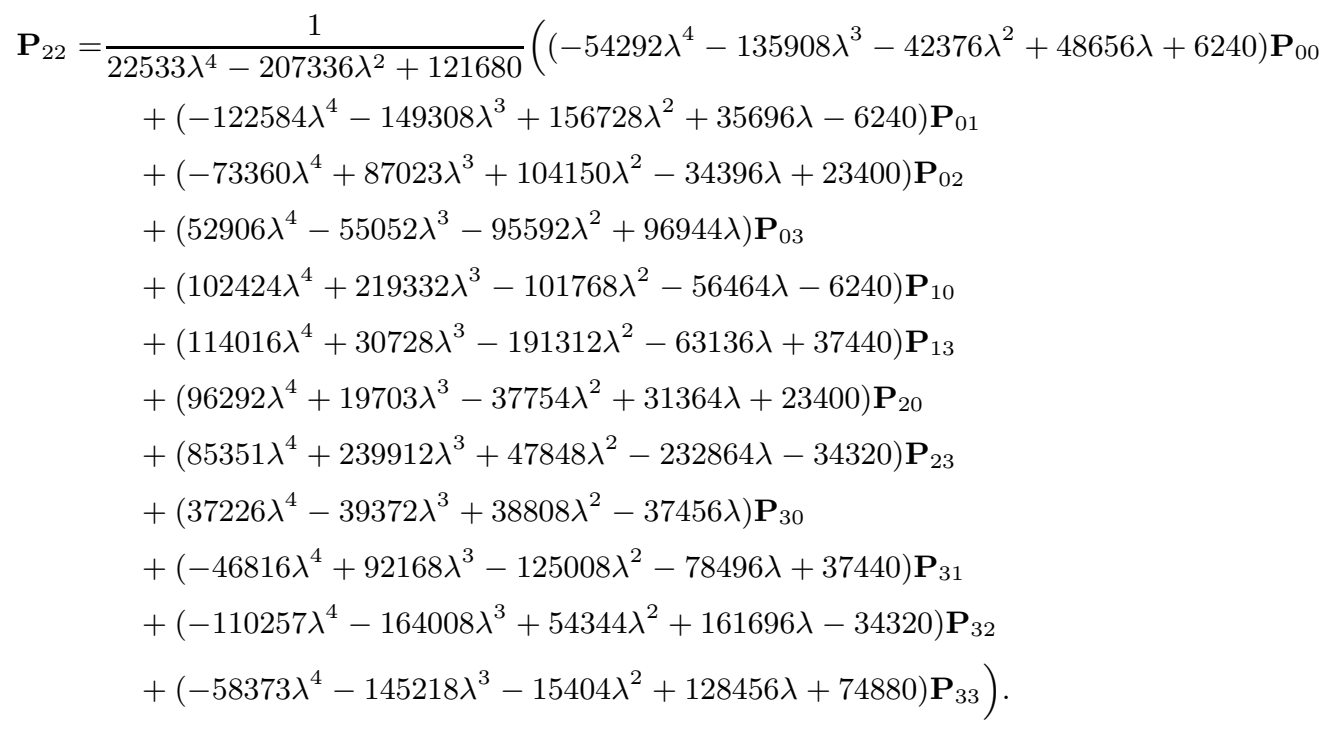




\section{A Comparative Study}

In this section, we discuss a study we have undertaken to compare among the weak-area functional method, the quasi-harmonic method [21], Dirichlet method [12], harmonic and biharmonic method [14] and the strain energy-minimizing method [11].
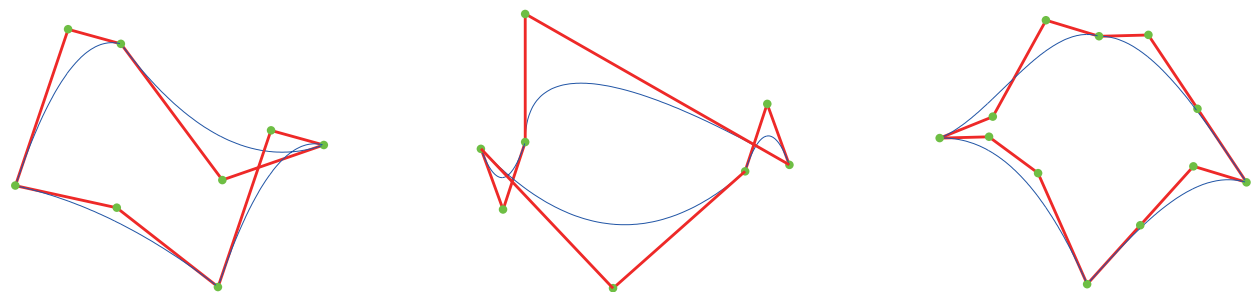

(a) Boundary curves for Example 1. (b) Boundary curves for Example 2. (c) Boundary curves for Example 3.
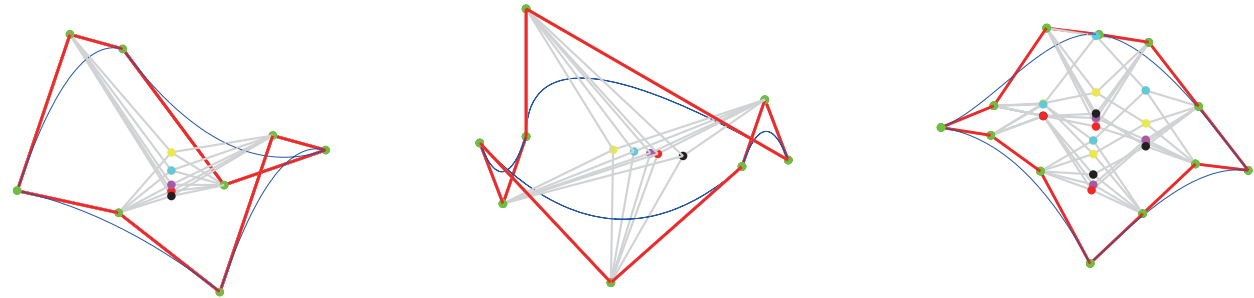

(d) Control points for Example 1.

(e) Control points for Example 2 .

(f) Control points for Example 3.

Fig. 4.1. Given boundary curves and control points of Examples 1-3 by different mask methods.

In [6], the authors introduced the following mask form:

$$
\begin{array}{lll}
\alpha & \beta & \alpha \\
\beta & \bullet & \beta \\
\alpha & \beta & \alpha
\end{array}
$$

with $4 \alpha+4 \beta=1$, denoted by $M_{\alpha}\left(\mathbf{P}_{i j}\right)$ in [12]. Following this idea, the quasi-harmonic mask can be denoted as $M_{\frac{19}{44}}\left(\mathbf{P}_{i j}\right)$, the Dirichelt mask is $M_{\frac{3}{8}}\left(\mathbf{P}_{i j}\right)$, the harmonic mask is $M_{\frac{1}{4}}\left(\mathbf{P}_{i j}\right)$ and the bending mask from biharmonic functional is $M_{\frac{1}{11}}\left(\mathbf{P}_{i j}\right)$. Compared with above mask methods, the linear relation between one inner control point and its eight neighboring control points from the extremal of the weak-area functional is

$$
\begin{array}{cccc}
-3 & -3-5 \lambda & 1-10 \lambda & 5 \lambda-3 \\
-\frac{10 \lambda+1}{8} \times & \bullet & 10 \lambda+1 \\
5 \lambda-3 & 1-10 \lambda & -3-5 \lambda
\end{array} .
$$

Obviously, the mask of the weak-area functional has no symmetry as $M_{\alpha}\left(\mathbf{P}_{i j}\right)$ unless $\lambda=0$, i.e., the Dirichlet energy.

Since the minimal surface in the Plateau problem has both the minimal area and zero mean curvature, as a means of checking the surface quality and making qualitative comparisons, we compare these methods with both area and average mean curvature in the approximation of the Plateau-Bézier problem. 

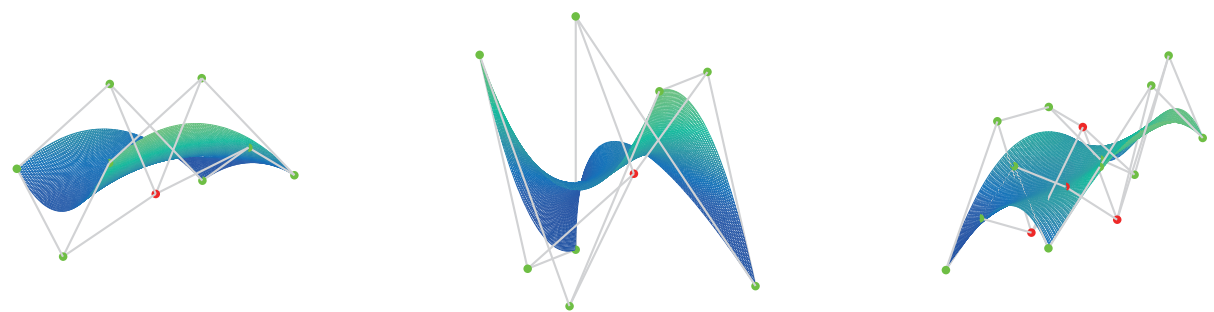

(a) quasi-harmonic mask for Example 1.

(b) quasi-harmonic mask for Example 2. (c)

(c) quasi-harmonic mask for Example 3.
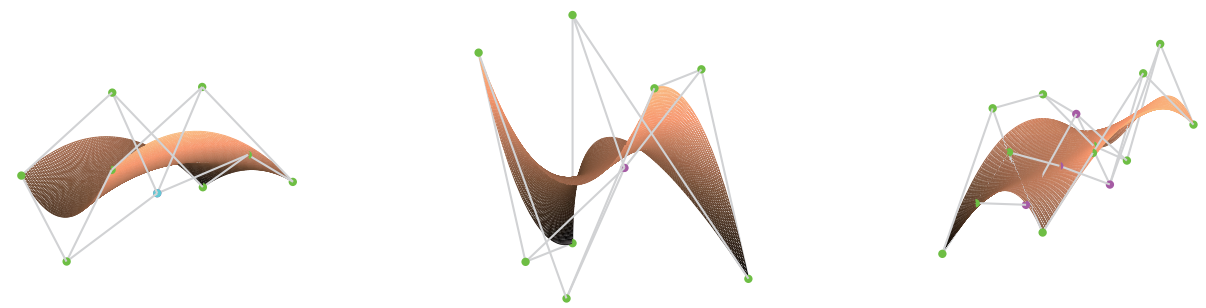

(d) Dirichlet method for Example 1. (e) Dirichlet method for Example 2. (f) Dirichlet method for Example 3.

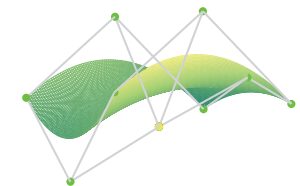

(g) $\lambda=0.04$ for Example 1 .

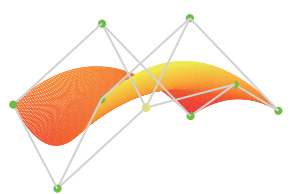

(j) harmonic mask for Example 1.

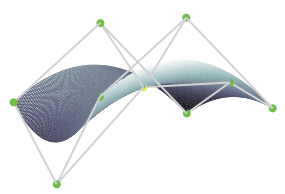

(m) bending mask for Example 1.

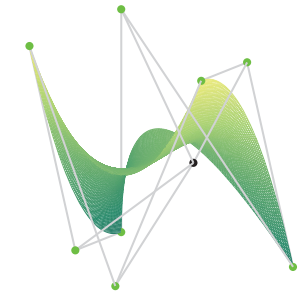

(h) $\lambda=-0.2$ for Example 2 .

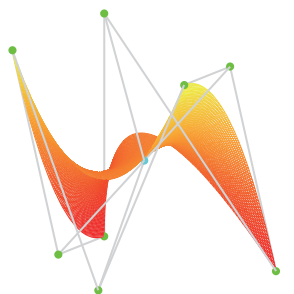

(k) harmonic mask for Example 2. (l) biharmonic mask for Example 3.
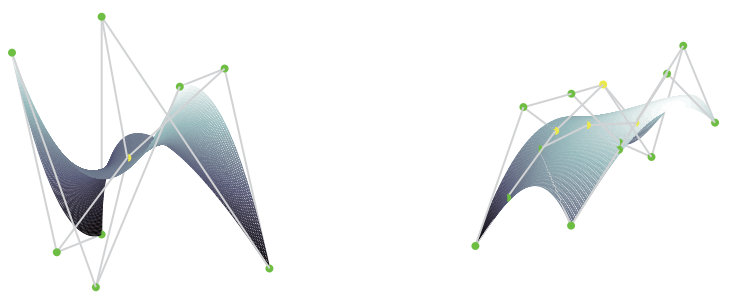

(n) bending mask for Example 2 .

(o) bending mask for Example 3 .

Fig. 4.2. Examples 1-3 by different mask methods. 
Table 4.1: Comparison among different methods for Example 1. AVMC: the average value of mean curvature at the $50 \times 50$ sampling points on the corresponding Bézier surface. The underline values are the minimal absolute values in the corresponding column.

\begin{tabular}{|c|cc|}
\hline Method & Area & AVMC \\
\hline quasi-harmonic & 5.6758 & 0.4839 \\
\hline Dirichlet & 5.6790 & 0.5422 \\
\hline harmonic & 5.6947 & 0.6696 \\
\hline strain energy-minimizing & 5.7313 & 0.8321 \\
\hline$\lambda=0.04$ & $\underline{5.6750}$ & $\underline{0.4392}$ \\
\hline
\end{tabular}

Table 4.2: Same as Table 4.1, especially for Example 2.

\begin{tabular}{|c|cc|}
\hline Method & Area & AVMC \\
\hline quasi-harmonic & 4.4950 & -0.3048 \\
\hline Dirichlet & 4.4954 & -0.3130 \\
\hline harmonic & 4.4968 & -0.3316 \\
\hline strain energy-minimizing & 4.4994 & -0.3568 \\
\hline$\lambda=-0.2$ & $\underline{4.4945}$ & $\underline{-0.2779}$ \\
\hline
\end{tabular}

Examples 1-3. In order to compare the different masks, three comparison examples are shown in Figs. 4.1-4.2. We first fix the four boundary curves with its control points, then construct the surface by computing the inner control point through the weak-area functional, the Dirichlet mask, the quasi-harmonic mask, the bending energy mask, the harmonic and biharmonic mask respectively. Different colors of the inner control points are used to represent different methods. Fig. 4.1 gives the different boundary curves and the comparison of corresponding control points obtained from different mask methods. Fig. 4.2 shows the comparison of the corresponding approximate minimal Bézier surfaces from different methods. The corresponding area values and mean curvature information are listed in Tables 4.1-4.3. For these examples, we can find that the proposed weak-area method can achieve the smallest area value and the smallest absolute average value of mean curvature by choosing appropriate parameters.

Table 4.3: Same as Table 4.1, especially for Example 3.

\begin{tabular}{|c|cc|}
\hline Method & Area & AVMC \\
\hline quasi-harmonic & 10.3375 & 0.2173 \\
\hline Dirichlet & 10.3155 & 0.5518 \\
\hline biharmonic & 11.3223 & 0.5621 \\
\hline strain energy-minimizing & 10.6965 & 0.7963 \\
\hline$\lambda=0.1$ & $\underline{10.3139}$ & $\underline{0.2148}$ \\
\hline
\end{tabular}

Remark 4.1. For the above examples one could note that, in some cases, the method resulting in the smallest surface area is for the extremal Bézier surfaces of weak-area functional with negative $\lambda$. It is also noteworthy that the results obtained by the methods presented here for the examples are better than those obtained by the minimisation of the Dirichlet or harmonic functionals. This is indeed true when isothermal parameterization condition is present in the energy functional. 


\section{Conclusion}

In this paper, we propose a new method to solve the Plateau-Bézier problem. Considering the importance of isothermal parameterization in the construction of minimal surface, we present a new energy functional called weak-area functional by combining the Dirichlet functional and weak isothermal parameterization condition. For some cases of given boundary Bézier curves, compared with the previous methods, Bézier surface with smaller area and mean curvature absolute values can be achieved by using the proposed weak-area energy functional with appropriate parameter $\lambda$. Several modeling examples show the efficiency of the proposed method.

Similarly, we can also construct a new energy functional by combining quasi-harmonic functional and weak isothermal parameterization, and obtain the corresponding results. In the future, we plan to obtain a better characterization of the isothermal parameterization, thus constructing a better approximation of the area functional to solve the numerical approximation problem of minimal surface.

Acknowledgments. This work was supported by the National Natural Science Foundation of China (Nos. 11801225, 11526098), University Science Research Project of Jiangsu Province (No. 18KJB110005) and the Research Foundation for Advanced Talents of Jiangsu University (No. 14JDG034).

\section{References}

[1] KA. Brakke, The surface evolver, Exp. Math., 1:2 (1992), 141-165.

[2] C. Coppin, D. Greenspan, A contribution to the particle modeling of soap films, Appl. Math. Comput., 26:4 (1988), 315-331.

[3] J. Douglas, A method of numerical solution of the problem of Plateau, Ann. Math., 29:(1-4) (1927/28) 180-188.

[4] J. Douglas, Solution of the problem of Plateau, Trans. Amer. Math. Soc., 33 (1931), 263-321.

[5] G. Dziuk, J. E. Hutchinson, The discrete Plateau problem: algorithm and numerics, Math. Comp., 68:225 (1999), 1-23.

6] G. Farin, D. Hansford, Discrete Coons patches, Comp. Aided Geom. Des., 16 (1999), 691-700.

[7] Y.X. Hao, C.J. Li, R.H. Wang, An approximation method based on MRA for the quasi-Plateau problem, BIT Numer. Math., 53 (2013), 411-442.

[8] Y.X. Hao, R-H. Wang, C.J. Li, Minimal quasi-Bézier surface, Appl. Math. Model, 36:2 (2012), $5751-5757$.

[9] M. Hinata, M. Shimasaki, T. Kiyono, Numerical solution of Plateau's problem by a finite element method, Math. Comput., 28:125 (1974), 45-60.

[10] K. Jung, K.T. Chu, S. Torquato, A variational level set approach for surface area minimization of triply-periodic surfaces, J. Comput. Phys, 2 (2007), 711-730.

[11] Y. Miao, H. Shou, J. Feng, Q. Peng, A.R. Forrest, Bézier surfaces of minimal internal energy, In IMA conference on the mathematics of surfaces, 2005, pp. 318-335.

[12] J. Monterde, Bézier surfaces of minimal area: The Dirichlet approach, Comp. Aided Geom. Des., 21 (2004), 117-136.

[13] J. Monterde, The Plateau-Bézier problem, In: Wilson, M. J., Martin, R.R. (eds.) Mathematics of Surfaces. LNCS, Springer, Heidelberg, 2768 (2003), 262-273.

[14] J. Monterde, H. Ugail, On harmonic and biharmonic Bézier surfaces, Comp. Aided Geom. Des., 7 (2004), 697-715.

[15] J.C.C. Nitsche, Lectures on minimal surfaces, vol.1. Cambridge Univ. Press, Cambridge, 1989. 
[16] R. Osserman, A survey of minimal surfaces, Dover publ. 2nd ed., New York, 1986.

[17] Q. Pan, G. Xu, Construction of minimal subdivision surface with a given boundary, Comp. Aided. Des., 43 (2011), 374-380.

[18] C.H. Séquin, CAD tools for aesthetic engineering, Comp. Aided. Des., 7 (2005), 737-750.

[19] Ø. Tråsdahl, E.M.Rønquist, High order numerical approximation of minimal surfaces, J. Comp. Phys, 230 (2011), 4795-4810.

[20] Y. Wang, Periodic surface modeling for computer aided nano design, Comp. Aided. Des., 3 (2007), $179-189$

[21] G. Xu, T. Rabczuk, E. Güler, Q. Wu, K. Hui, G. Wang, Quasi-harmonic Bézier approximation of minimal surfaces for finding forms of structural membranes, Comput. Struct., 161 (2015), 55-63.

[22] G. Xu, Y. Zhu, G. Wang, A. Galligo, L. Zhang, K. Hui, Explicit form of parametric polynomial minimal surfaces with arbitrary degree, Appl. Math. Comput., 259 (2015), 124-131.

[23] G. Xu, G. Wang, Quintic parametric polynomial minimal surfaces and their properties, Differ. Geom. Appl., 28:6 (2010), 697-704.

[24] Q. Zhang, G.L. Xu, $G^{2}$ surface modeling using minimal mean curvature variation flow, Comp. Aided. Des., 39:5 (2007), 342-351. 